\title{
Solvent-Adsorbate Interactions and Adsorbate-Specific Solvent Structure in Carbon Dioxide Reductionon a Stepped Cu Surface
}

Ludwig, Thomas; Gauthier, Joseph A.; Brown, Kristopher S.; Ringe, Stefan; Nørskov, Jens K.; Chan, Karen

Published in:

Journal of Physical Chemistry C

Link to article, DOI:

10.1021/acs.jpcc.8b11571

Publication date:

2019

Document Version

Peer reviewed version

Link back to DTU Orbit

Citation (APA):

Ludwig, T., Gauthier, J. A., Brown, K. S., Ringe, S., Nørskov, J. K., \& Chan, K. (2019). Solvent-Adsorbate Interactions and Adsorbate-Specific Solvent Structure in Carbon Dioxide Reductionon a Stepped Cu Surface. Journal of Physical Chemistry C, 123(10), 5999-6009. https://doi.org/10.1021/acs.jpcc.8b11571

\section{General rights}

Copyright and moral rights for the publications made accessible in the public portal are retained by the authors and/or other copyright owners and it is a condition of accessing publications that users recognise and abide by the legal requirements associated with these rights.

- Users may download and print one copy of any publication from the public portal for the purpose of private study or research.

- You may not further distribute the material or use it for any profit-making activity or commercial gain

- You may freely distribute the URL identifying the publication in the public portal 


\section{Solvent-Adsorbate Interactions and}

\section{Adsorbate-Specific Solvent Structure in Carbon Dioxide Reduction on a Stepped Cu Surface}

Thomas Ludwig, ${ }^{\dagger, \ddagger}$ Joseph A. Gauthier, ${ }^{\dagger, \ddagger}$ Kristopher S. Brown, ${ }^{\dagger, \ddagger}$ Stefan Ringe, ${ }^{\dagger, \ddagger}$ Jens K. Nørskov, ${ }^{\llbracket}$ and Karen Chan ${ }^{*}, \mathbb{9}$

$\dagger$ SUNCAT Center for Interface Science and Catalysis, SLAC National Accelerator Laboratory, 2575 Sand Hill Road, Menlo Park, California 94025, United States $\ddagger S U N C A T$ Center for Interface Science and Catalysis, Department of Chemical Engineering, Stanford University, Stanford, California 94305, United States ФDepartment of Physics, Technical University of Denmark, 2800 Kongens Lyngby, Denmark

E-mail: kchan@fysik.dtu.dk 


\begin{abstract}
In this work, the structure of water and its interactions with various carbon dioxide reduction intermediates adsorbed on a $\mathrm{Cu}(211)$ surface is investigated using density functional theory. We find that the presence of adsorbates has a significant and adsorbate-specific effect on the local water structure, and that solvation can stabilize adsorbate conformations different than those found in vacuum. We describe relationships between the hydrogen bonding capability of an adsorbate, the dipole moment of the adsorbate, the energetic strength of water-adsorbate interactions, and the change induced in the local water orientation by the adsorbate. Mechanistic implications are discussed. We investigate and quantify the error associated with using arbitrary locally optimized solvent structures in calculations of relevant physical quantities, such as solvated binding energies and work functions. Possible effects of thermal motion on calculations of the work function are investigated using ab initio molecular dynamics.
\end{abstract}

\title{
Introduction
}

The electrolyte is a critical part of electrochemical systems. It facilitates the separation of the oxidation and reduction half-reactions within the electrochemical cell, conducts ions, and directly interacts with the reaction intermediates at the electrochemical interface. Experimentally, it has been demonstrated that many properties of the electrolyte, such as $\mathrm{pH},{ }^{1,2}$ solvent concentration, ${ }^{3,4}$ transport properties $^{5-7}$ and specific ion concentrations ${ }^{8,9}$ significantly affect selectivity and activity of various electrocatalytic processes. A systematic understanding of electrolyte effects would facilitate the rational design of the electrolyte as an integral part of the optimization of electrochemical processes. Theoretical and computational approaches play an important role towards this end, given the experimental challenges associated with investigating reaction processes in situ and operando. ${ }^{10,11}$

The structure of water on metal surfaces has been studied for many years. ${ }^{12,13}$ Experiments on close-packed transition metal surfaces such as $\mathrm{Pt}(111)$ using a variety of x-ray 
spectroscopic methods ${ }^{14}$ as well as $\mathrm{Pd}(111)$ and $\mathrm{Ru}(0001)$ using scanning tunneling microscopy (STM) ${ }^{15}$ reveal a predominantly hexagonal, ice-like structure under vacuum conditions and low temperatures. Theoretical investigations of these hexagonal structures using density functional theory (DFT) revealed that on more reactive surfaces such as $\mathrm{Ru}(0001)$, the surface water layer may be partially dissociated, ${ }^{16}$ while an intact structure without dissociation was predicted on $\operatorname{Pt}(111) .{ }^{14}$ The simple ice-like descriptions have since been called into question ${ }^{17}$ and a diverse collection of water structures on flat metal surfaces has been described. ${ }^{18-22}$ It has also been observed experimentally using STM that water binds more strongly to low coordination sites such as step edges on $\mathrm{Pt}^{23}$ and $\mathrm{Cu},{ }^{24}$ leading to additional features compared to flat surfaces. Experimental investigations of oxide ${ }^{25}$ and bimetallic $^{26}$ surfaces have characterized the effects of various surface heterogeneities on water structure. There is also experimental evidence that adsorbed species can have specific effects on the water structure. $^{27}$

The many experimental observations have prompted theoretical investigations on water structure on elemental metals, ${ }^{17,18,28-35}$ oxides, ${ }^{25,36-40}$ bimetallic alloys, ${ }^{26,41}$ and stepped transition metal facets. ${ }^{32,42-44}$ Recent theoretical work has provided a systematic understanding of of how various surface properties affect surface binding of water. ${ }^{45}$ Several studies have also investigated the effects of explicitly including $\mathrm{pH} .{ }^{40,46,47}$ Although the reaction thermochemistry is often investigated without the explicit consideration of the electrolyte, ${ }^{48}$ it has been demonstrated that ions can significantly affect the thermodynamics due to field effects. ${ }^{49-51}$ Calculations of electrochemical reaction barriers generally must also explicitly consider at least the reacting ions, which usually requires some amount of explicit solvation. ${ }^{52-54}$ For this reason, many studies have investigated the effect of including explicit solvent structures and electric field effects in calculations on reaction mechanisms and kinetics. ${ }^{8,9,49-51,53-66}$ Of the studies including explicit electrolyte species, some use structures which are locally optimized, representing local minima in the potential energy surface, while others employ $a b$ initio molecular dynamics simulations to sample thermodynamic ensembles at realistic tem- 
peratures. The drawback of using only local minima is that the possible effects of thermal fluctuations are not explicitly included. However, using ab initio molecular dynamics simulations can be orders of magnitude more computationally expensive than studying locally optimal structures. In both cases, it is difficult to determine whether relevant configurations have been sufficiently sampled. Even with molecular dynamics, the simulation lengths used are often on the order of approximately 10-100 ps, which may not be long enough to sufficiently sample statistically independent configurations due to the correlation time scales for water relaxation near metal interfaces at room temperature, which may be on the order of tens of picoseconds or longer. ${ }^{67,68}$

Electrolyte effects can be modeled using a wide variety of atomistic and continuum approaches. In general, classical force fields and continuum implicit solvation models allow for the exploration of significantly larger system sizes at much reduced computational cost. There are several challenges involved in accurately modeling a metal-water interface using classical molecular dynamics, including the implementation of an ideally polarizable metal model which includes the detailed electrostatic induction effects in the metal, specific adsorption, and constant electrode potential. ${ }^{67-69}$ Although these challenges have been addressed in several applications of classical force fields to metal-water interfaces, ${ }^{67-71}$ the use of such classical force field methods involves the careful implementation of sophisticated models which may be less readily transferable to new systems than ab initio models. Implicit solvation has also been successfully applied to the study of water-metal interfaces. Although methods such as joint density functional theory ${ }^{72}$ and reference interaction site model $^{73}$ are capable of calculating the equilibrium structure of solvent in combination with DFT calculations, most implicit solvent methods used in combination with $\mathrm{DFT}^{74-77}$ by design do not provide finely detailed structural information about the solvent at atomic resolution. Understanding atomic-level detail in the solvation structure can provide important understanding of how the specific solvation structure may affect energetics and mechanisms.

In this study we use DFT and an explicit solvent model of the metal-water interface cou- 
pled with a global optimization algorithm to study the structure of water and its interactions with adsorbates on a stepped $\mathrm{Cu}(211)$ surface. We focus on a set of key adsorbed intermediates relevant to the $\mathrm{CO}_{2}$ and $\mathrm{CO}$ reduction reactions. ${ }^{50,57,78}$ We apply a constrained minima hopping algorithm ${ }^{79,80}$ to thoroughly sample the local minima inherent to the potential energy surface of the solvated systems. We characterize the water structure near $\mathrm{Cu}(211)$ and how it is changed by the presence of adsorbates, and we describe systematic relationships between these structural changes and the energetics associated with the adsorbate-water interactions. We quantify errors associated with the use of explicit solvent in DFT calculations by quantitatively comparing physical quantities of the global minima with those associated with statistics from the distributions of local minima results. We also consider the possible effect of thermal motion on calculations of the work function.

\section{Computational Details}

Density functional calculations were performed using the Quantum ESPRESSO electronic structure suite, ${ }^{81}$ using the Atomic Simulation Environment (ASE) as a user interface. ${ }^{82}$ The dispersion-corrected Bayesian Error Estimation Functional BEEF-vdW ${ }^{83-86}$ was used as the exchange-correlation functional. The Vanderbilt ultra-soft pseudopotentials ${ }^{87-90}$ were used to represent the core electrons, and the valence electrons were expanded in a plane wave basis, and the Brillouin zone was sampled using a Monkhorst Pack (MP) k-point grid. ${ }^{91}$ Details are provided in the Supporting Information (SI).

Global optimization of the water structures was carried out using a modified version of the constrained minima hopping algorithm. ${ }^{79,80}$ It should be emphasized that application of minima hopping does not guarantee that the global minimum configuration will be found. Moreover, the very large number of potential local minima in systems of this size, ${ }^{79,92}$ combined with the computational expense of DFT, complicates determining convergence of the results relative to number of iterations. This is further discussed in the SI. Ab initio molec- 
ular dynamics calculations were performed at constant temperature, number of particles, and volume (NVT) as implemented in ASE. ${ }^{82}$ The hydrogen bonding interactions were investigated using an analysis based on the bond orders as calculated using properties of the charge density with Chargemol and GPAW. ${ }^{93-96}$ The hydrogen bonds drawn in the atomistic visualization figures were produced using simple geometric criteria validated by comparison with the bond order analysis. ${ }^{97}$ Details are provided in the SI.

\section{Results and Discussion}

\section{Water Structure on Clean Cu(211) Surface}

We investigated the structure of water on clean $\mathrm{Cu}(211)$ surfaces with 5 and $10-\mathrm{H}_{2} \mathrm{O}$ per supercell, containing 9 surface atoms per simulation supercell. Figure 1 shows the structures corresponding to the global minima. A single water molecule per supercell adsorbs oxygendown on the step edge. Since the supercell contains 3 step edge sites, the coverage on the step edge sites is $1 / 3$. This low coverage of the step edge results from the competition between water-water and water-surface interactions. Although the interaction of the water monomer with the step edge is favorable, the hydrogen bonding network favors a structure which promotes binding to sites other than step edges. An implication of this relevant to catalysis is that adsorption of a reaction intermediate on a step edge does not require the displacement or desorption of any step-adsorbed water, since $2 / 3$ of the step sites are vacant. Higher energy, less stable structures with $2 / 3$ step edge water coverages were also found, and the most stable of these structures and energies are provided in the SI, but no stable structures with full water coverage on the step edge were found. 


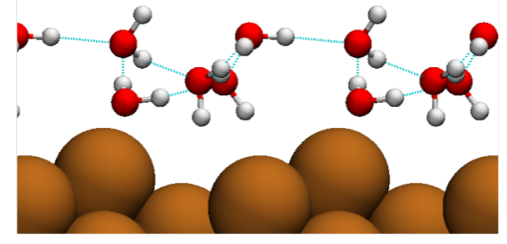

a

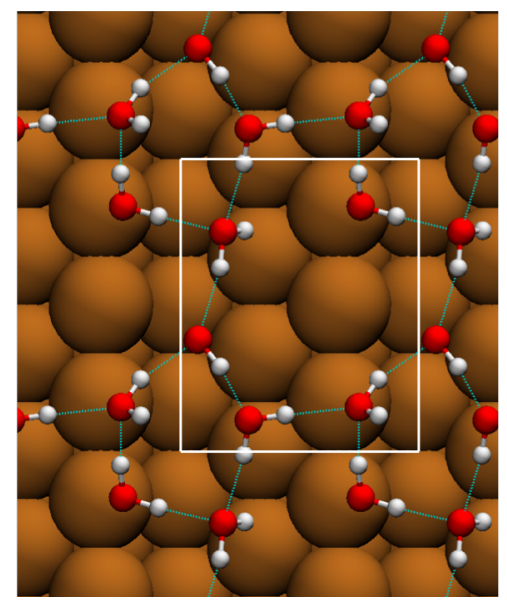

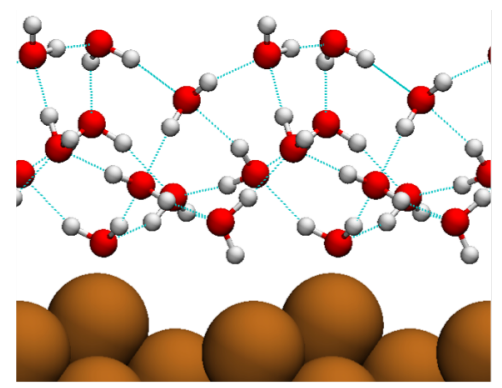

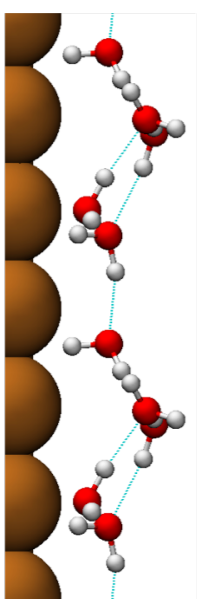

b

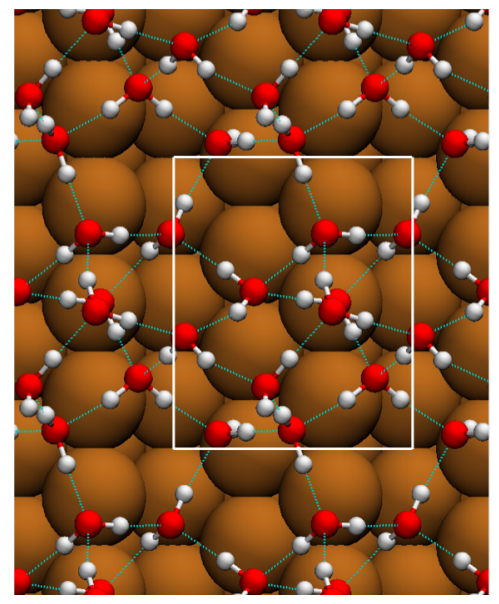

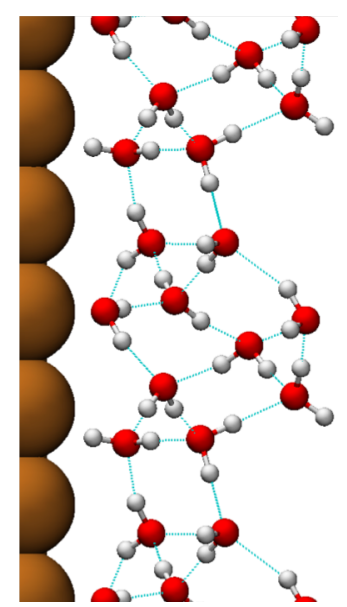

Figure 1: Global optimal structures of water on clean $\mathrm{Cu}(211)$. a: $5-\mathrm{H}_{2} \mathrm{O}$ per supercell. b: $10-\mathrm{H}_{2} \mathrm{O}$ per supercell). The three panels show the projection of the structure in three orthogonal directions. The white rectangle indicates the periodic boundaries of the supercell. 
Figure 1 shows that $\mathrm{H}_{2} \mathrm{O}$ is oriented hydrogen-down at terraces and hydrogen-up at stepedge sites. On the solvated $\mathrm{Cu}(211)$ surface, the step edges carry a small partial positive charge where $\mathrm{H}_{2} \mathrm{O}$ is adsorbed hydrogen-up, while the terrace sites carry a small partial negative charge. Results of Bader charge analysis are shown in the SI. The hydrogen bonding network is dominated by repeating pentagonal motifs. The oxygen-down adsorption at step edges, hydrogen-down interactions at terrace sites, and presence of pentagonal rings resemble results reported in a recent joint experimental-DFT study on $\mathrm{Cu}(511) .{ }^{42}$ This suggests that step features on facets other than 211 can have a similar effect on the interfacial water structure, and that the results reported in this work can be expected to extend to various types of $\mathrm{Cu}$ step sites.

In the most stable $10-\mathrm{H}_{2} \mathrm{O}$ configuration, also displayed in Figure 1, a similar structure near the surface is observed. The coverage adjacent to the surface remains similar to that used in the $5-\mathrm{H}_{2} \mathrm{O}$ calculations. This optimal structure illustrates that the water beyond the interfacial layer participates readily in hydrogen bonding with the interfacial water layer, in contrast to the hydrophobic interfacial layer described near some more strongly binding metal surfaces. ${ }^{67,68}$ This connectivity of the hydrogen bond network may be of relevance to proton transport mechanisms near the surface. A five-membered or pentagonal hydrogenbonded ring structure is present in the $10-\mathrm{H}_{2} \mathrm{O}$ per supercell configuration, as in the $5-\mathrm{H}_{2} \mathrm{O}$

per supercell configuration. The preferential oxygen-down orientation at approximately $1 / 3$ of the step edges and hydrogen-down orientation at terrace sites described for the $5-\mathrm{H}_{2} \mathrm{O}$ per supercell results is retained with the additional water molecules present.

\section{Solvation Effects on Binding Energies}

The solvated binding energy $\Delta G_{\text {bind }}^{\text {solv }}$ of an adsorbate can be expressed as the sum of two contributions:

1. energy of cavity formation $\Delta G_{\text {cav }}$ : the energy associated with reorganization of solvent from its relaxed equilibrium configuration with no adsorbate present into the solvation 
shell configuration which it assumes in the presence of a given adsorbate, and

2. energy of solvated adsorbate insertion $\Delta G_{\text {ins }}$ : the energy associated with insertion of the adsorbate from the gas phase into its minimum energy configuration, bound to the surface, within the already-configured equilibrium solvent shell.

The solvation energy $\Delta G_{\text {solv }}$ is the difference between the binding energies in solvent and vacuum, as written in equation 1.

$$
\Delta G_{\mathrm{solv}}=\Delta G_{\mathrm{bind}}^{\mathrm{solv}}-\Delta G_{\mathrm{bind}}^{\mathrm{vac}}=\Delta G_{\mathrm{cav}}+\left[\Delta G_{\mathrm{ins}}-\Delta G_{\mathrm{bind}}^{\mathrm{vac}}\right]
$$

Defining a "pre-organized solvation energy" $\Delta G_{\mathrm{solv}}^{\mathrm{pre}}$ as the term enclosed by brackets in equation 1, we decompose the solvation energy into two convenient terms,

$$
\Delta G_{\mathrm{solv}}=\Delta G_{\mathrm{cav}}+\Delta G_{\mathrm{solv}}^{\mathrm{pre}}
$$

where $\Delta G_{\text {solv }}^{\text {pre }}$ quantifies the strength of the interaction between the adsorbate and the already-organized optimal solvation shell. Quantifying the energies associated with solvent

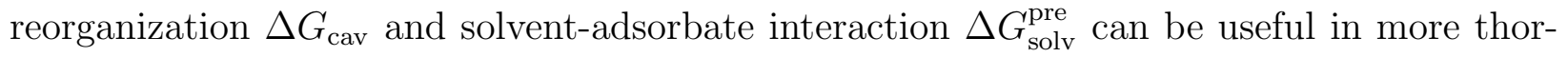
oughly understanding the solvation of adsorbed intermediates.

The effect of water on a few key elementary reaction energies relevant to $\mathrm{CO}_{2}$ reduction catalysis was evaluated by comparing the reaction energies obtained including the explicit solvent in the model to the energies obtained in vacuum. The decomposition of solvation energy according to equation 2 is obtained by 'single point' energy calculations on structures derived from the full local minima structures by deleting specific atoms while holding all other atoms fixed, as described in the SI. The results for all adsorbates considered are summarized in Figure 2. The effects on the reaction free energy diagram are presented later in Figure 4. A noticeable yet small difference between 5 and $10-\mathrm{H}_{2} \mathrm{O}$ models is seen only in the larger $\mathrm{C}_{2}$ adsorbates $\mathrm{OCCO}$ and $\mathrm{OCCHO}$, which due to their size may extend further into the solvent 
phase than the smaller adsorbates do.
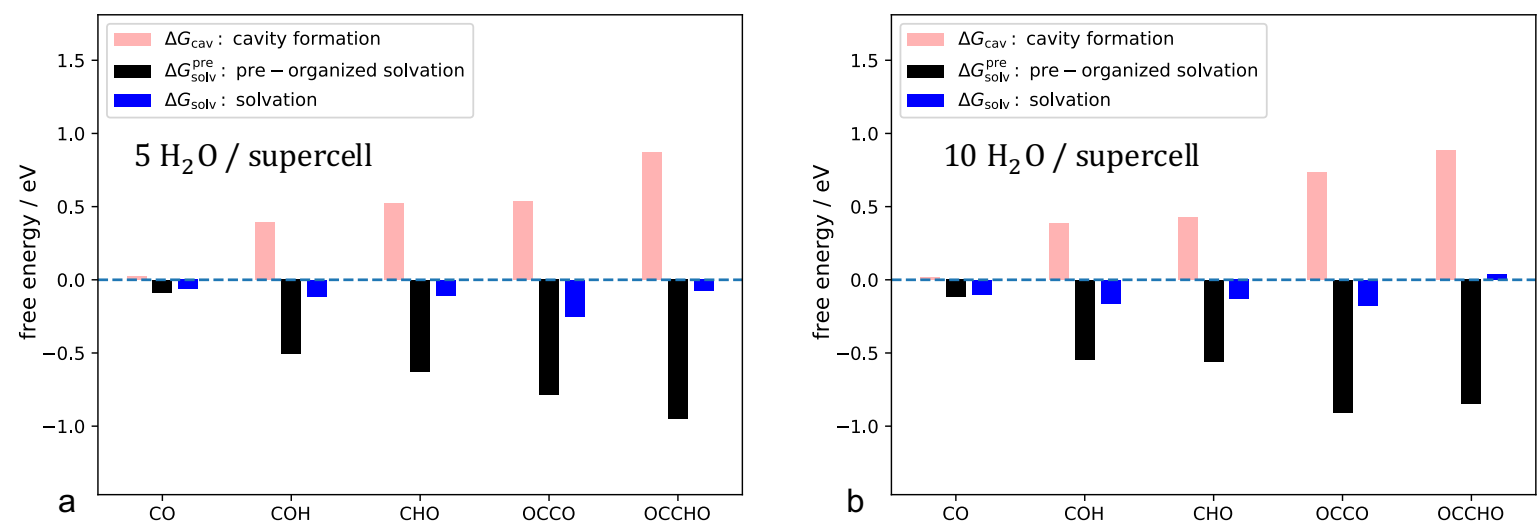

Figure 2: Contributions to solvation energy of adsorbed intermediates. a: $5-\mathrm{H}_{2} \mathrm{O} /$ supercell. b: $10-\mathrm{H}_{2} \mathrm{O} /$ supercell). All quantities are Boltzann-weighted averages.

The energetic cost of organizing the solvent into the optimal solvation shell can be quite large, on the order of 0.5 to $1 \mathrm{eV}$. The large cavity formation energy is evidence that adsorbates can induce significant changes in the local structure of water. The energetic cost of these structural changes scales closely with the energetic favorability of the adsorbate interactions with the new adsorbate-induced solvation structure. There is thus a significant cancellation between the cavity formation and insertion energies. More energetically favorable interactions between the adsorbate and the water (more negative $\Delta G_{\text {solv }}^{\text {pre }}$ ) are correlated with more extensive restructuring of the water near the adsorbate (more positive $\Delta G_{\text {cav }}$ ). The trends in $\Delta G_{\text {cav }}$, and $\Delta G_{\text {solv }}^{\text {pre }}$ for the $5-\mathrm{H}_{2} \mathrm{O}$ and $10-\mathrm{H}_{2} \mathrm{O}$ configurations are similar. Trends in $\Delta G_{\text {solv }}$ are more subtle and difficult to confidently assess due to their smaller size, especially since the magnitudes of errors in DFT calculations for nonbonded interactions are on the order of $0.1 \mathrm{eV}{ }^{83}$ The magnitude of the solvation energy roughly increases with adsorbate size in the $5-\mathrm{H}_{2} \mathrm{O}$ calculations, but this trend is not as clear in the $10-\mathrm{H}_{2} \mathrm{O}$ case, and OCCHO appears to be an exception to this trend in both cases. The physical significance of the energetic trends is more thoroughly explored in Figure 3 and the subsequent discussion. 


\section{Adsorbate-Specific Solvation Structure}

The presence of adsorbates induces changes in the local solvent structure relative to that on the clean stepped $\mathrm{Cu}$ surface, as illustrated in Figure 3(a). More detailed images of the global minimal structures are available in the SI, as are direct comparisons of the $5-\mathrm{H}_{2} \mathrm{O}$ and $10-\mathrm{H}_{2} \mathrm{O}$ results.

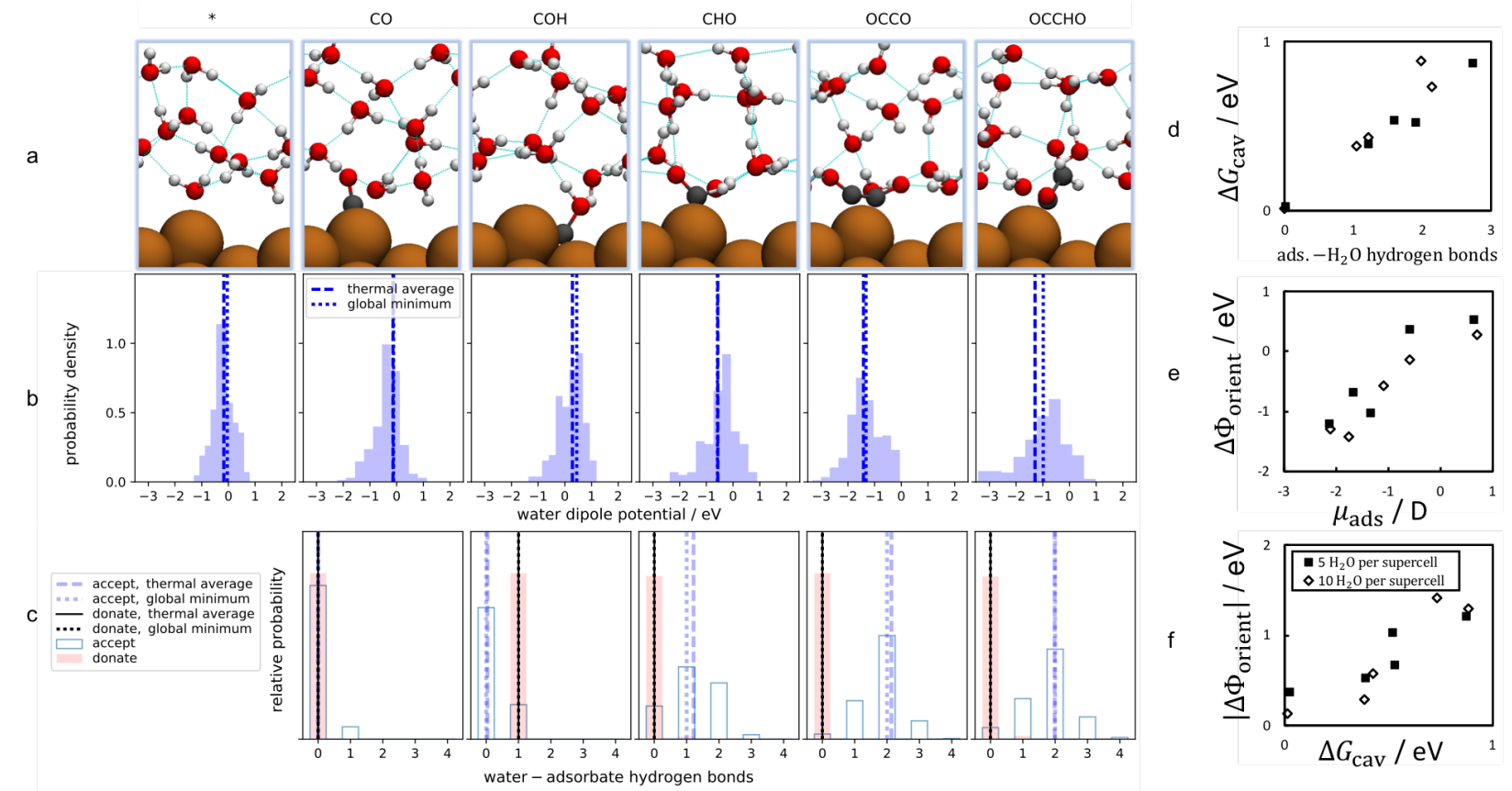

Figure 3: Analysis of solvation structure. a: Illustration of global minima structures. b: Global minimum, Boltzmann-weighted average, and unweighted local minima distribution of water dipole potential $\Delta \Phi_{\text {orient }}$. c: Global minimum, Boltzmann-weighted average, and unweighted local minima distribution of hydrogen bonding characteristics. d: cavity formation energy $\Delta G_{\text {cav }}$ versus total hydrogen bonds for all adsorbates considered. e: Water dipole potential $\Delta \Phi_{\text {orient }}$ versus adsorbate vacuum dipole moment $\mu_{\text {ads }}$ in Debye for all adsorbates considered. f: Absolute value of the water dipole potential versus the cavity formation energy for all adsorbates considered. All quantities in d., e., and f. are Boltzmann-weighted averages over all local minima. Only the $10-\mathrm{H}_{2} \mathrm{O}$ data is shown in b. and c., and comparisons to $5-\mathrm{H}_{2} \mathrm{O}$ data are in the SI.

The global minimal structures displayed in Figure 3(a) suggest that hydrogen bonding can be significant contributor to adsorbate-water interactions. In particular, $\mathrm{COH}$ donates a hydrogen bond to a nearby water, while $\mathrm{CHO}, \mathrm{OCCO}$, and $\mathrm{OCCHO}$ all accept at least one hydrogen bond and do not appear to donate any. The $\mathrm{CO}$ adsorbate does not participate in 
hydrogen bonding to any appreciable extent. This is consistent with the observation from Figure 2 that $\mathrm{CO}$ interacts quite weakly with the solvent.

The results of a more quantitative analysis of the hydrogen bonding characteristics is displayed in Figure 3(c). The bars in Figure 3(c) constitute histograms of the unweighted distributions of hydrogen bond numbers in all the local minima. The unfilled bars indicate the number of hydrogen bonds accepted by the adsorbate and the filled bars indicate number of hydrogen bonds donated by the adsorbate in each local minimum configuration. The vertical lines overlayed on these histograms indicate the calculated values of the Bolztmannweighted averages over all local minima, as well as the values at the global minima, of the number of hydrogen bonds accepted by and donated by each adsorbate as indicated in the legend. The agreement between the results evaluated at the global minima and the Boltzmann-weighted averages over all local minima indicates that the global minima structures shown are representative of the Boltzmann-weighted average results. The correlation between $\Delta G_{\text {cav }}$ and the total number of adsorbate-water hydrogen bonds, plotted in Figure $3(\mathrm{~d})$, indicates that the energetic cost of solvent restructuring in response to the presence of the adsorbate scales with the extent of hydrogen bonding between the solvent and the adsorbate.

To quantify how the presence of adsorbates affects the local orientation of water, we calculate the electrostatic potential energy change across the isolated water molecules in the absence of the metal surface and the adsorbate. We refer to this here as the 'water dipole potential' $\Delta \Phi_{\text {orient }}$ where the subscript emphasizes that it is the orientation contribution only, consistent with previous work involving this quantity. ${ }^{98-100}$ This quantifies the effect of water orientation on the work function of the surface, neglecting the polarization contribution. The dominant contribution to this potential drop is the dipole moment density of the water molecules. A positive water dipole potential as defined here corresponds to a net hydrogendown structure, while a negative water dipole potential corresponds to a net hydrogen-up structure. 
The structures associated with clean $\mathrm{Cu}(211)$ as well as adsorbed $\mathrm{CO}$ show nearly zero net water dipole orientation. The result for clean $\mathrm{Cu}(211)$ is consistent with previous studies of water near metal electrodes. ${ }^{29,34}$ The observation that CO induces only a very small change in the local water orientation is once again consistent with its very weak interaction with the solvent (Figure 2). In contrast, the other adsorbates induce significant changes in the local water orientation. $\mathrm{COH}$ induces a positive water dipole potential, while $\mathrm{CHO}$, OCCO, and $\mathrm{OCCHO}$ induce negative water dipole potentials. This indicates that $\mathrm{COH}$ induces a net hydrogen-down structure, while $\mathrm{CHO}, \mathrm{OCCO}$, and $\mathrm{OCCHO}$ induce net hydrogenup structures, at the coverages studied. A correlation between the water dipole potential and the adsorbate vacuum dipole moment is apparent in Figure 3 (e). This means that the average dipole orientation induced in the water is antiparallel to the direction of the adsorbate dipole. The most stable orientation of a dipole in response to the field created by another spatially fixed dipole depends on its position relative to the fixed dipole and its axis. Directly vertically above the fixed dipole along its axis, the most stable alignment is parallel, while in the plane bisecting the axis of the fixed dipole the most stable alignment is antiparallel. In this case, where the adsorbate can be thought of as having a fixed dipole and the water molecules are relatively free to reorient in response to its presence, there are more water molecules laterally beside the adsorbate on the surface than there are vertically directly above it, and thus on average the antiparallel alignment is stabilized. This suggests that the correlation described here is expected to be specific to this particular adsorbate coverage, and could change as adsorbate coverages increases.

The magnitude of the water dipole potential is correlated with the magnitude of the cavity formation energy, as shown in Figure 3 (f). Coupled with the correlation between the adsorbate dipole moment and the water dipole poential, this suggests that the electrostatic interaction between the dipole moment of the adsorbate and the local water structure has a role in the restructuring of the water in response to the presence of an adsorbate. Note that in these plots $\Delta G_{\text {solv }}^{\text {pre }}$ and $\Delta G_{\text {cav }}$ could be used essentially interchangeably except for a 
sign change, since those quantities are also correlated (Figure 2). Together, the correlation plots in Figure 3(d-f) suggest that the local solvent structure restructures in response to the presence of an adsorbate by forming hydrogen bonds with the adsorbate and aligning with the field induced by the adsorbate dipole moment, and that the energy associated with this solvent restructuring $\Delta G_{\text {cav }}$ and with the resulting water-adsorbate interactions $\Delta G_{\text {solv }}^{\text {pre }}$ both scale with these structural descriptors.

\section{Mechanistic Implications}

The specificity of the local water structure to each adsorbed intermediate suggests that elementary reaction steps, such as proton transfers and C-C coupling, are accompanied by rearrangements in the local water structure. This adsorbate-specific solvation structure may affect the energetic barriers associated with relevant elementary reaction steps in several ways. Even if solvation does not significantly change the relative stabilities of the initial and final states of an elementary step, it could have an effect on the kinetics if significant reorganization is required due to different solvation structures in the initial and final states, since the solvent re-orientation energy barrier contributes to the barrier associated with the elementary step.

As an example of the possible mechanistic significance of solvent re-organization, we consider two possible C-C coupling reactions: $2 * \mathrm{CO} \rightarrow * \mathrm{OCCO}$ and $* \mathrm{CO}+* \mathrm{CHO} \rightarrow * \mathrm{OCCHO}$. In Figure 3(b,c), it is clear that both *OCCO and *OCCHO induce hydrogen-up local water structures and accept approximately two hydrogen bonds. Examining the reactants, ${ }^{*} \mathrm{CO}$ is associated with a neutral water structure and accepts no hydrogen bonds, while ${ }^{*} \mathrm{CHO}$ induces a hydrogen-down local water structure and accepts one or two hydrogen bonds. Thus, the solvation structure of ${ }^{*} \mathrm{CHO}$ is quantitatively similar to that of the corresponding final state, ${ }^{*} \mathrm{OCCHO}$, while the solvation structure of ${ }^{*} \mathrm{CO}$ is quite different than that of the corresponding final state, ${ }^{*} \mathrm{OCCO}$, in hydrogen bonding, orientation, and cavity formation energy. This suggests that the water re-organization contribution to the barrier may 
be greater in $\mathrm{CO}-\mathrm{CO}$ coupling than in $\mathrm{CO}-\mathrm{CHO}$ coupling, and this solvent re-organization contribution would promote $\mathrm{C}-\mathrm{C}$ coupling of ${ }^{*} \mathrm{CHO}$ relative to ${ }^{*} \mathrm{CO}$. Previous computational work has indicated a lower activation barrier for C-C coupling with increasing hydrogenation of the reactants even when explicit solvent is not considered, ${ }^{78}$ and the present results indicate that the effects of solvent re-organization could also contribute to this trend.

Thermal fluctuations in the solvation structure could likewise influence the subsequent elementary reaction steps by pre-solvation, which can play an important role in the rates of many aqueous processes, including proton transfer, ${ }^{101-103}$ in an analogous manner to the role of solvent reorganization in electron transfer reactions. ${ }^{104}$ For example, when instantaneous thermal fluctuation of the water structure near adsorbed CO cause a momentarily hydrogen-up structure, then this pre-organized solvation structure may promote the formation of $\mathrm{CHO}$ via proton transfer relative to $\mathrm{COH}$, since $\mathrm{CHO}$ is stabilized by a locally hydrogen-up solvation structure while $\mathrm{COH}$ is stabilized by a locally hydrogen-down solvation structure. It is also plausible that proton transfers to the surface are more facile from predominantly hydrogen-down water structures, while a predominantly hydrogen-up water structure could increase this barrier since a water molecule must rotate before the proton can be transferred. A shortcoming of this study is that the explicit solvent effects on the transition state structures are not studied, and the dynamic effects of pre-organization and reorganization on reaction events are not directly studied. This motivates future work focused on understanding the kinetic effects of the local electrolyte structure.

\section{Comparison of Global Minima to Local Minima Distributions}

In addition to searching for a global minimal structure, the minima-hopping configuration sampling procedure finds a diverse collection of local minima. ${ }^{80}$ In this work the minima hopping procedure was continued until the distributions of energy from this sampling method were observed to relatively converge as described in the SI. Assuming that the collection of local minima found in this procedure is roughly representative of the collection of inherent 
local minima in the potential energy surface of the system, we can compare results obtained using statistics of this collection to those evaluated at the global minima. This is of practical importance because many DFT calculations which include explicit solvent structures do not employ exhaustive global minimization or configuration sampling procedures, as was done in this work. A comparison of results obtained from statistics of the representative local minima distribution to those obtained from global minimization is a step towards quantifying the accuracy and precision of using solvent structures which have not been thoroughly globally minimized. Of primary interest are the free energies and the work functions, which are shown in Figures 4 and 5, respectively. We emphasize that this error analysis is associated with the computational explicit-solvent model itself, rather than a comparison to experimentally measured quantities.
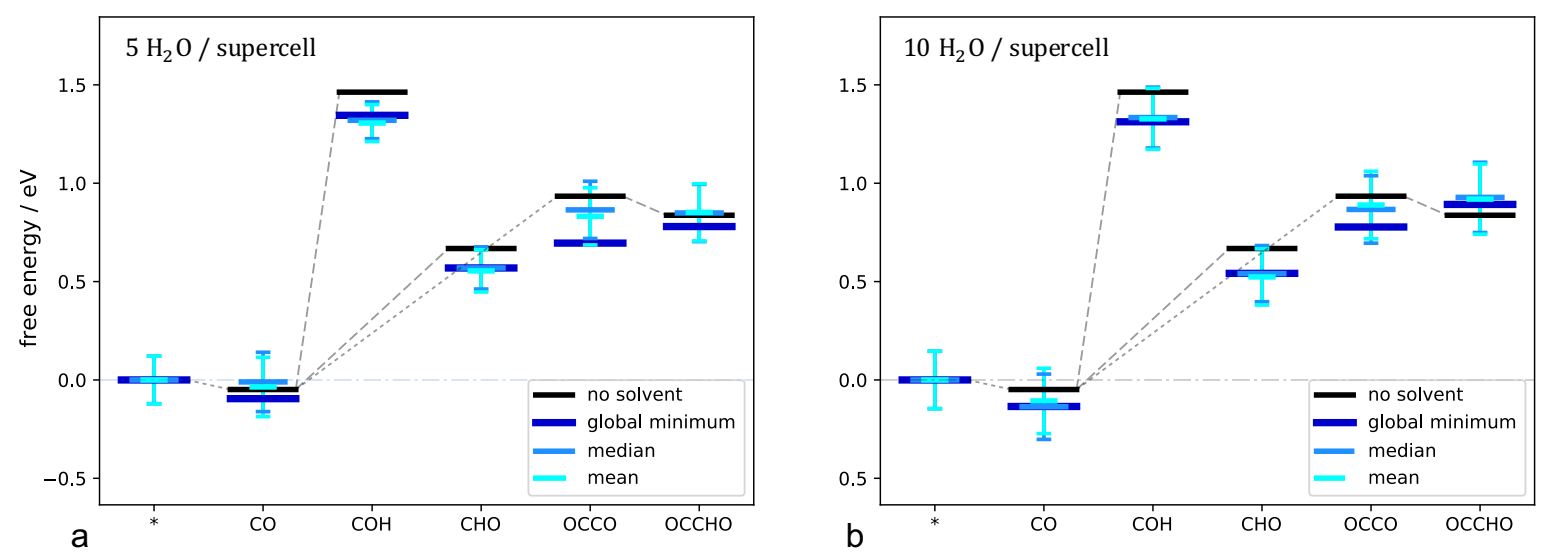

Figure 4: Comparison of free energies of adsorbed intermediates calculated from the global minima structures to those computed from statistics of the local minima distributions. The error bars quantify one standard deviation of the results computed from the local minima distributions.

There is a distribution of possible calculated free energies for each adsorbate which arises from the collection of possible solvation structures. Figure 4 illustrates that the expectation values of these distributions are quite close to the physically relevant values given by the global minima. For $\mathrm{CO}, \mathrm{COH}$, and $\mathrm{CHO}$, the results found using the median or arithmetic mean of the local minima distributions are nearly indistinguishable from those from the 
global minima. For OCCO and OCCHO, using the median or mean tends to underpredict the effect of solvation, resulting in a slightly less stable energy than does the global minimum result. The largest error is in the prediction of the OCCO solvated binding energy, for which the error is approximately $0.15 \mathrm{eV}$ in the $5-\mathrm{H}_{2} \mathrm{O}$ per supercell case and approximately 0.1 $\mathrm{eV}$ in the $10-\mathrm{H}_{2} \mathrm{O}$ per supercell case. In all other cases, the difference between the median and mean results and the global minima results is less than $0.1 \mathrm{eV}$. The bias for the clean solvated surface is zero here because the corresponding solvated surface is always used as the reference for the binding energies. The mean and median operations are also applied to these reference states. This means that the reference energies corresponding to the median and mean structures are greater than the reference energies corresponding to the global minima. This is why the binding energies calculated from the local minima distributions can be lower than those calculated from the global minima.

Convergence of the expectation values of the local minima results to the physically correct values is practically useful only if the variances of these distributions are also relatively small, such that a small number of samples drawn from this collection could yield a reasonable result. The error bars in Figure 4 indicate the positions of one standard deviation above and below the given statistic on the free energy diagram. This is equivalent to approximately a $68 \%$ prediction interval for a given free energy if only a single structure is sampled, at random, from the collection of possible local minima. This quantifies the expected deviation from the global minimum result caused by using an arbitrarily chosen solvation structure. For the $5-\mathrm{H}_{2} \mathrm{O}$ per supercell case, these standard deviations are on the order of 0.1 to $0.15 \mathrm{eV}$, while for the $10-\mathrm{H}_{2} \mathrm{O}$ per supercell case, the standard deviations are slightly higher, but always less than $0.2 \mathrm{eV}$. This suggests that as more water molecules are included, the variance introduced into the calculation by using an arbitrary local minimum solvent structure increases.

A similar analysis can be applied to the work function, which is often used in DFT calculations to quantify the absolute electrode potential. ${ }^{52,105}$ Figure 5 displays the distribution of work functions from the collection of local minima structures for each adsorbed interme- 
diate. Except in the case of $\mathrm{CO}$ with $5-\mathrm{H}_{2} \mathrm{O}$ per supercell, the expectation values of these distributions are quite close to the work functions of the global minimum structures.

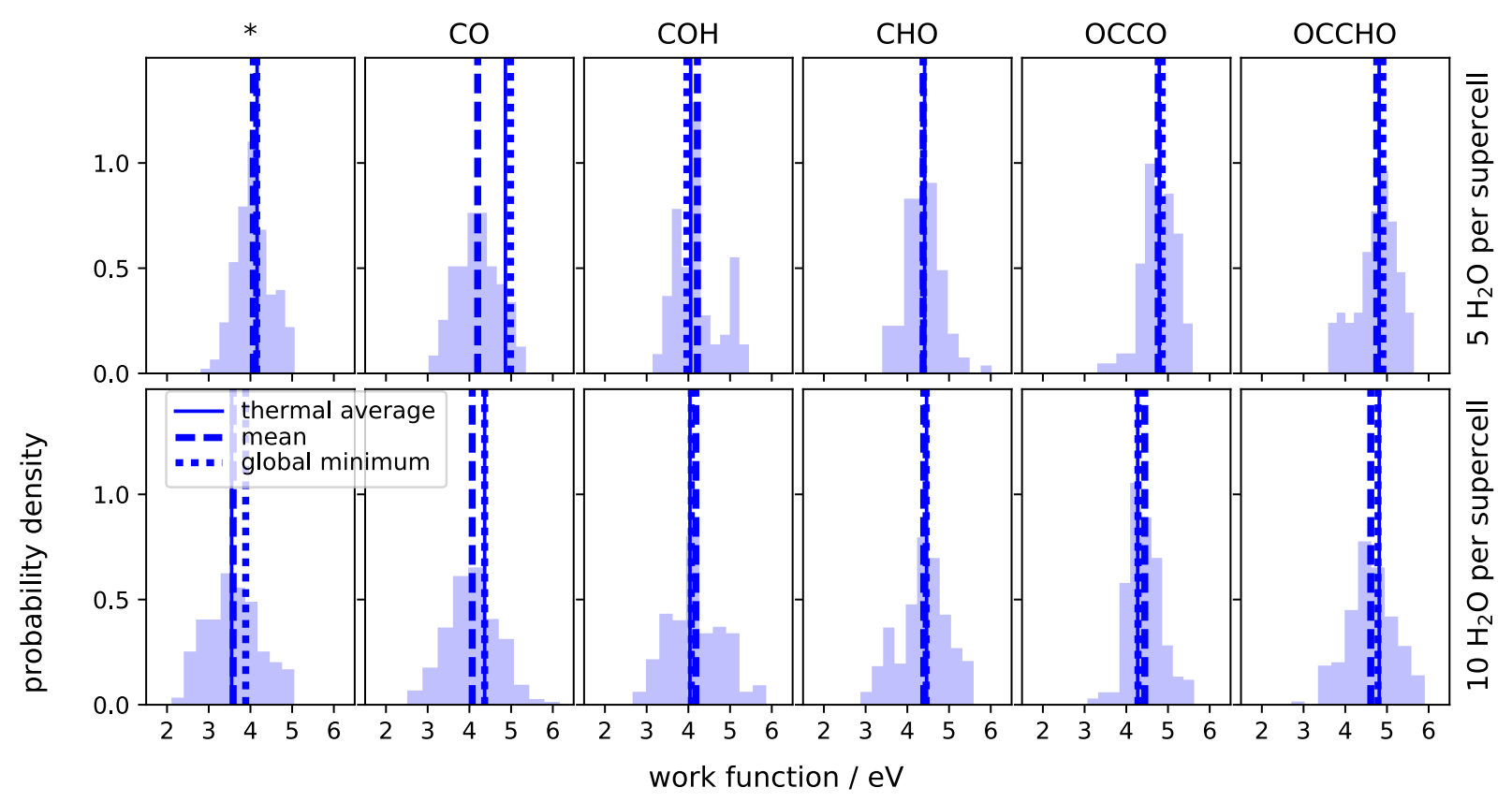

Figure 5: Comparison of work functions of solvated $\mathrm{Cu}(211)$ with adsorbed intermediates calculated from the global minima structures to those computed from the local minima distributions.

The correspondence between the expectation values of the local minima distributions and the global minima results indicates that the work function at the global minimum structure may be approximated by sampling a number of local minima from a representative sample. In practice, since the variance is quite large, the work function of a single arbitrarily chosen local minimum structure is often quite different from that at the global minimum. This underscores the importance of sufficiently sampling solvent configuration when the physical quantity of interest depends very sensitively on the details of the solvent structure, as is the case with the work function. ${ }^{98}$

Finally, we consider again the hydrogen bonding results shown in Figure 3(c). The unweighted distributions of hydrogen bonding characteristics from the entire collections of local minima sampled reveal that the higher-energy local minima also generally display 
hydrogen bonding characteristics qualitatively similar to those of the global minima: the adsorbates with global minima that accept hydrogen bonds have local minima collections which predominantly accept hydrogen bonds, and those with global minima that donate hydrogen bonds have local minima collections which predominantly donate hydrogen bonds. This is of practical importance because it means that structural conclusions drawn from the study of arbitrarily chosen local minima may often be consistent with those drawn from rigorously optimized global minima structures.

\section{The Effect of Thermal Motion on the Work Function}

The results discussed in the previous sections have focused on the local minima in the potential energy surface of the solvent structure. As the temperature is increased from $0 \mathrm{~K}$ to more experimentally relevant temperatures, thermal excitations or fluctuations away from these local minima become increasingly important. To evaluate the possible effects of thermal motion on the structure, we performed short ab initio molecular dynamics simulations starting from three arbitrarily chosen distinct local minima of the clean surface with $5 \mathrm{H}_{2} \mathrm{O}$ per supercell and no adsorbate. For each of these molecular dynamics simulations, the work function recorded at each time step. Each system was equilibrated with a thermostat set at $300 \mathrm{~K}$ for $500 \mathrm{fs}$. After equilibration, the simulation was continued for another 1500 fs,

during which equilibrium statistics were recorded. Details are provided in the SI. The work function results are summarized in Figure 6. 


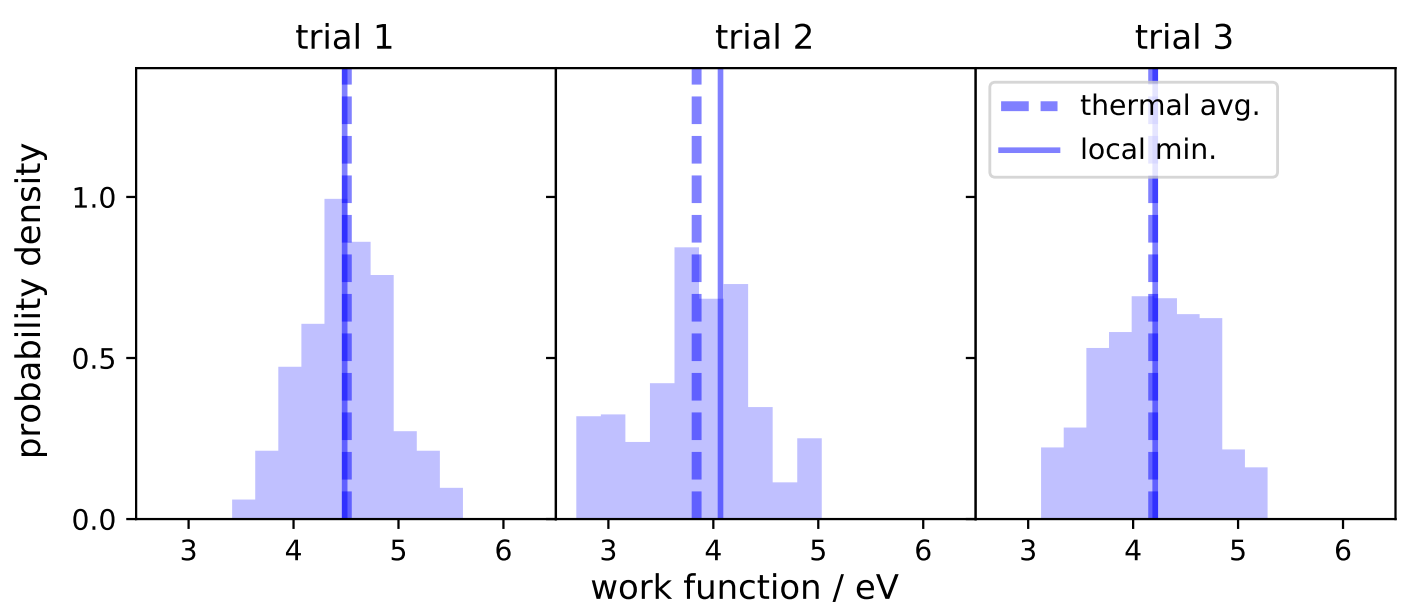

Figure 6: Comparison of work functions at local minima to ensemble averages and fluctuations from ab initio molecular dynamics simulations.

For all three trials, the time-averaged work function is comparable to the work function of the starting local minimum. This suggests that at time scales on the order of picoseconds, the time-averaged work function associated with a trajectory started from a given local minimum is similar to the work function evaluated at the corresponding local minimum. Therefore, there does not appear to be a significant systematic error in the work function calculation introduced by using its value at a local minimum structure rather than its ensemble average value at a nonzero temperature. On the other hand, this initial investigation indicates that thermal fluctuations can be substantial and motivates a more thorough investigation of thermal motion in future work.

The relative fluctuations are expected to be sensitive to the size of the simulated system. Here the relevant dimension is the geometric surface area of the electrochemical interface per supercell, which in this case is approximately $49 \AA^{2}$. These molecular dynamics simulations were carried out on clean $\mathrm{Cu}(211)$ with five rigid water molecules per supercell. We also emphasize here that understanding the accuracy and applicability of DFT for the structure and properties of liquid water is also an active research area. ${ }^{34,106-110}$ These considerations motivate further study of the effects of thermal energy on solvation structure near adsorbates. 


\section{Conclusions}

In this work, the solvent structure near adsorbed carbon dioxide reduction intermediates on $\mathrm{Cu}(211)$ was investigated using DFT calculations coupled with a global minimization algorithm. We find that each adsorbate has a significant and unique effect on the local water structure. We quantify and systematically explain these adsorbate-specific structural changes by considering the relationships between the adsorbate-water hydrogen bonding, adsorbate dipole moment, the change in water orientation induced by the adsorbate, and the energetic strength of water-adsorbate interactions. Polar adsorbates can accept or donate hydrogen bonds and induce a net dipole moment orientation change locally in the solvent. The magnitude of this orientation change scales with the energetic cost associated with the organization of the solvent to the presence of an adsorbate, which in turn is closely correlated with the interaction strength between the water and the adsorbate. This analysis of adsorbate-specific solvation structure represents a step towards a systematic understanding of how changes in solvation structure interact with elementary reaction steps at electrochemical interfaces, providing initial mechanistic insight and directions for future work.

By comparing results obtained from global minima structures to results found by sampling the local minima distributions, we quantify the accuracy and precision associated with the common practice of using arbitrary locally optimal solvent structures without employing a thorough global minimization procedure. We find that the the solvation energies calculated from the global minima structures can be reproduced with reasonable accuracy (the largest error is approximately $0.15 \mathrm{eV}$, and most errors are less than $0.1 \mathrm{eV}$ ) and reasonably small variance using arbitrarily sampled local minima. The standard deviation of the local minima results is found to be on the order of $0.15 \mathrm{eV}$ for 5 to 10 explicitly included water molecules. This roughly quantifies the error introduced in studies which use explicit solvent structures without thorough global minimization procedures. Comparisons to experimentally measured solvation energetics are not made here, and the error analysis is associated with the computational method itself. 
We find that the work function corresponding to the global minima structures can also be reproduced by sampling arbitrary local minima, but in this case the variance is quite large due to the sensitive dependence of the work function on the detailed solvent structure. This indicates that for quantities which depend sensitively on the specific details of the solvent structure, thorough configurational sampling is crucial to ensure physically meaningful results. The variance in work function also motivates the future investigation of solvent dynamics using hybrid continuum-atomistic models or other controlled work function methods.

In our investigation of the possible effects of thermal motion on the water structure using $a b$ initio molecular dynamics, we find that at short time scales on the order of 2 ps the timeaverage of the work function is relatively close to the work function at the corresponding local minimum, while the substantial fluctuations observed provide motivation for future work to more comprehensively study these effects.

\section{Acknowledgement}

Support from the U.S. Department of Energy, Office of Basic Energy Science, to the SUNCAT Center for Interface Science and Catalysis is gratefully acknowledged. This work was supported by the U.S. Department of Energy, Office of Science, Office of Basic Energy Science, via Grant DE-SC0008685 to the SUNCAT Center of Interface Science and Catal-

ysis. T.L. acknowledges support from the United States Department of Energy through the Computational Sciences Graduate Fellowship (DOE CSGF) under grant number DEFG02-97ER25308. K.S.B. was supported by the Department of Defense (DoD) through the National Defense Science \& Engineering Graduate Fellowship (NDSEG) Program.

\section{Supporting Information Available}

All data plotted in figures, computational details, convergence tests, hydrogen bonding analysis, Bader charge analysis, detailed images and descriptions of adsorbate structures, com- 
parisons of 5 and $10-\mathrm{H}_{2} \mathrm{O}$ per supercell results, details of single point calculations, free energy corrections and other corrections and calculations used.

This material is available free of charge via the Internet at http://pubs.acs.org/.

\section{References}

(1) Strmcnik, D.; Uchimura, M.; Wang, C.; Subbaraman, R.; Danilovic, N.; Van Der Vliet, D.; Paulikas, A. P.; Stamenkovic, V. R.; Markovic, N. M. Improving the Hydrogen Oxidation Reaction Rate by Promotion of Hydroxyl Adsorption. Nature chemistry 2013, 5, 300 .

(2) Ledezma-Yanez, I.; Wallace, W. D. Z.; Sebastián-Pascual, P.; Climent, V.; Feliu, J. M.; Koper, M. T. Interfacial Water Reorganization as a pH-dependent Descriptor of the Hydrogen Evolution Rate on Platinum Electrodes. Nature Energy 2017, 2, 17031.

(3) Tomita, Y.; Teruya, S.; Koga, O.; Hori, Y. Electrochemical Reduction of Carbon Dioxide at a Platinum Electrode in Acetonitrile-Water Mixtures. Journal of The Electrochemical Society 2000, 147, 4164-4167.

(4) Tsuneto, A.; Kudo, A.; Sakata, T. Lithium-Mediated Electrochemical Reduction of High Pressure N2 to NH3. Journal of Electroanalytical Chemistry 1994, 367, 183-188.

(5) Yoon, Y.; Hall, A. S.; Surendranath, Y. Tuning of Silver Catalyst Mesostructure Promotes Selective Carbon Dioxide Conversion Into Fuels. Angewandte Chemie 2016, $128,15508-15512$.

(6) Wuttig, A.; Yaguchi, M.; Motobayashi, K.; Osawa, M.; Surendranath, Y. Inhibited Proton Transfer Enhances Au-Catalyzed CO2-to-Fuels Selectivity. Proceedings of the National Academy of Sciences 2016, 113, E4585-E4593.

(7) Seidel, Y.; Schneider, A.; Jusys, Z.; Wickman, B.; Kasemo, B.; Behm, R. Mesoscopic 
Mass Transport Effects in Electrocatalytic Processes. Faraday discussions 2009, 140, $167-184$.

(8) Resasco, J.; Chen, L. D.; Clark, E.; Tsai, C.; Hahn, C.; Jaramillo, T. F.; Chan, K.; Bell, A. T. Promoter Effects of Alkali Metal Cations on the Electrochemical Reduction of Carbon Dioxide. Journal of the American Chemical Society 2017, 139, 1127711287.

(9) Perez-Gallent, E.; Marcandalli, G.; Figueiredo, M. C.; Calle-Vallejo, F.; Koper, M. T. Structure- and Potential-Dependent Cation Effects on CO Reduction at Copper SingleCrystal Electrodes. Journal of the American Chemical Society 2017, 139, 1641216419.

(10) Dou, J.; Sun, Z.; Opalade, A. A.; Wang, N.; Fu, W.; Tao, F. F. Operando Chemistry of Catalyst Surfaces During Catalysis. Chemical Society Reviews 2017, 46, 2001-2027.

(11) Ogasawara, H.; Kaya, S.; Nilsson, A. Operando X-Ray Photoelectron Spectroscopy Studies of Aqueous Electrocatalytic Systems. Topics in catalysis 2016, 59, 439-447.

(12) Thiel, P. A.; Madey, T. E. The Interaction of Water With Solid Surfaces: Fundamental Aspects. Surface Science Reports 1987, 7, 211-385.

(13) Henderson, M. A. The Interaction of Water With Solid Surfaces: Fundamental Aspects Revisited. Surface Science Reports 2002, 46, 1-308.

(14) Ogasawara, H.; Brena, B.; Nordlund, D.; Nyberg, M.; Pelmenschikov, A.; Pettersson, L. G. M.; Nilsson, A. Structure and Bonding of Water on Pt(111). Phys. Rev. Lett. 2002, 89, 276102.

(15) Tatarkhanov, M.; Ogletree, D. F.; Rose, F.; Mitsui, T.; Fomin, E.; Maier, S.; Rose, M.; Cerd, J. I.; Salmeron, M. Metal- And Hydrogen-Bonding Competition During Wa- 
ter Adsorption on $\mathrm{Pd}(111)$ and $\mathrm{Ru}(0001)$. Journal of the American Chemical Society 2009, 131, 18425-18434, PMID: 19947628.

(16) Feibelman, P. J. Partial Dissociation of Water on Ru (0001). Science 2002, 295, 99102.

(17) Hodgson, A.; Haq, S. Water Adsorption and the Wetting of Metal Surfaces. Surface Science Reports 2009, 64, 381-451.

(18) Carrasco, J.; Hodgson, A.; Michaelides, A. A Molecular Perspective of Water at Metal Interfaces. Nature materials 2012, 11, 667.

(19) Schiros, T.; Ogasawara, H.; Naslund, L.-Å.; Andersson, K. J.; Ren, J.; Meng, S.; Karlberg, G.; Odelius, M.; Nilsson, A.; Pettersson, L. G. Cooperativity in Surface Bonding and Hydrogen Bonding of Water and Hydroxyl at Metal Surfaces. The Journal of Physical Chemistry C 2010, 114, 10240-10248.

(20) McBride, F.; Hodgson, A. Water and Its Partially Dissociated Fragments at Metal Surfaces. International Reviews in Physical Chemistry 2017, 36, 1-38.

(21) Feibelman, P. J.; Kimmel, G. A.; Smith, R. S.; Petrik, N. G.; Zubkov, T.; Kay, B. D. A Unique Vibrational Signature of Rotated Water Monolayers on Pt (111): Predicted and Observed. The Journal of chemical physics 2011, 134, 204702.

(22) Carrasco, J.; Michaelides, A.; Forster, M.; Haq, S.; Raval, R.; Hodgson, A. A OneDimensional Ice Structure Built From Pentagons. Nature materials 2009, 8, 427.

(23) Morgenstern, M.; Michely, T.; Comsa, G. Anisotropy in the Adsorption of H2O at Low Coordination Sites on Pt(111). Physical review letters 1996, 77, 703.

(24) Yamada, T.; Tamamori, S.; Okuyama, H.; Aruga, T. Anisotropic Water Chain Growth on $\mathrm{Cu}$ (110) Observed With Scanning Tunneling Microscopy. Physical review letters 2006, 96, 036105. 
(25) Mu, R.; Zhao, Z.-j.; Dohnalek, Z.; Gong, J. Structural Motifs of Water on Metal Oxide Surfaces. Chem. Soc. Rev. 2017, 46, 1785-1806.

(26) McBride, F.; Darling, G. R.; Pussi, K.; Hodgson, A. Tailoring the Structure of Water at a Metal Surface: A Structural Analysis of the Water Bilayer Formed on an Alloy Template. Physical review letters 2011, 106, 226101.

(27) Kimmel, G. A.; Zubkov, T.; Smith, R. S.; Petrik, N. G.; Kay, B. D. Turning Things Downside Up: Adsorbate Induced Water Flipping on Pt (111). The Journal of chemical physics 2014, 141, 18C515.

(28) Bellarosa, L.; García-Muelas, R.; Revilla-Lopez, G.; Lopez, N. Diversity at the WaterMetal Interface: Metal, Water Thickness, and Confinement Effects. ACS central science 2016, 2, 109-116.

(29) Schnur, S.; Groß, A. Properties of Metal-Water Interfaces Studied from First Principles. New Journal of Physics 2009, 11, 125003.

(30) Michaelides, A.; Ranea, V. A.; de Andres, P. L.; King, D. A. General Model for Water Monomer Adsorption on Close-Packed Transition and Noble Metal Surfaces. Phys. Rev. Lett. 2003, 90, 216102.

(31) Michaelides, A. Density Functional Theory Simulations of Water-Metal Interfaces: Waltzing Waters, a Novel 2D Ice Phase, and More. Applied Physics A 2006, 85, $415-425$.

(32) Groß, A.; Gossenberger, F.; Lin, X.; Naderian, M.; Sakong, S.; Roman, T. Water Structures at Metal Electrodes Studied by Ab Initio Molecular Dynamics Simulations. Journal of The Electrochemical Society 2014, 161, E3015-E3020.

(33) Velasco-Velez, J.-J.; Wu, C. H.; Pascal, T. A.; Wan, L. F.; Guo, J.; Prendergast, D.; 
Salmeron, M. The Structure of Interfacial Water on Gold Electrodes Studied by X-Ray Absorption Spectroscopy. Science 2014, 1259437.

(34) Sakong, S.; Forster-Tonigold, K.; Groß, A. The Structure of Water at a Pt (111) Electrode and the Potential of Zero Charge Studied From First Principles. The Journal of Chemical Physics 2016, 144, 194701.

(35) Sakong, S.; Groß, A. The Electric Double Layer at Metal-Water Interfaces Revisited Based on a Charge Polarization Scheme. The Journal of Chemical Physics 2018, 149, 084705 .

(36) Gauthier, J. A.; Dickens, C. F.; Chen, L. D.; Doyle, A. D.; Nørskov, J. K. Solvation Effects for Oxygen Evolution Reaction Catalysis on IrO2(110). The Journal of Physical Chemistry C 2017, 121, 11455-11463.

(37) Cheng, J.; Sprik, M. Acidity of the Aqueous Rutile TiO2 (110) Surface From Density Functional Theory Based Molecular Dynamics. Journal of chemical theory and computation 2010, 6, 880-889.

(38) Cheng, J.; Sulpizi, M.; VandeVondele, J.; Sprik, M. Hole Localization and Thermochemistry of Oxidative Dehydrogenation of Aqueous Rutile TiO2 (110). ChemCatChem 2012, 4, 636-640.

(39) Sun, C.; Liu, L.-M.; Selloni, A.; Lu, G. Q. M.; Smith, S. C. Titania-Water Interactions: A Review of Theoretical Studies. Journal of Materials Chemistry 2010, 20, 1031910334 .

(40) Watanabe, E.; Rossmeisl, J.; Bjorketun, M. E.; Ushiyama, H.; Yamashita, K. AtomicScale Analysis of the RuO2 Water Interface Under Electrochemical Conditions. The Journal of Physical Chemistry C 2016, 120, 8096-8103. 
(41) Fischer, J. M.; Mahlberg, D.; Roman, T.; Groß, A. Water Adsorption on Bimetallic PtRu/Pt (111) Surface Alloys. Proc. R. Soc. A 2016, 472, 20160618.

(42) Lin, C.; Avidor, N.; Corem, G.; Godsi, O.; Alexandrowicz, G.; Darling, G. R.; Hodgson, A. Two-Dimensional Wetting of a Stepped Copper Surface. Phys. Rev. Lett. 2018, 120, 076101.

(43) Kolb, M. J.; Wermink, J.; Calle-Vallejo, F.; Juurlink, L. B.; Koper, M. T. Initial Stages of Water Solvation of Stepped Platinum Surfaces. Physical Chemistry Chemical Physics 2016, 18, 3416-3422.

(44) Kolb, M. J.; Farber, R. G.; Derouin, J.; Badan, C.; Calle-Vallejo, F.; Juurlink, L. B.; Killelea, D. R.; Koper, M. T. Double-Stranded Water on Stepped Platinum Surfaces. Physical review letters 2016, 116, 136101.

(45) Kakekhani, A.; Roling, L. T.; Kulkarni, A.; Latimer, A. A.; Abroshan, H.; Schumann, J.; AlJama, H.; Siahrostami, S.; Ismail-Beigi, S.; Abild-Pedersen, F. et al. Nature of Lone-Pair-Surface Bonds and Their Scaling Relations. Inorganic Chemistry 2018, 5\%, 7222-7238, PMID: 29863849.

(46) Hansen, M. H.; Nilsson, A.; Rossmeisl, J. Modelling pH and Potential in Dynamic Structures of the Water/Pt (111) Interface on the Atomic Scale. Physical Chemistry Chemical Physics 2017, 19, 23505-23514.

(47) Hansen, M. H.; Rossmeisl, J. pH in Grand Canonical Statistics of an Electrochemical Interface. The Journal of Physical Chemistry C 2016, 120, 29135-29143.

(48) Nørskov, J. K.; Rossmeisl, J.; Logadottir, A.; Lindqvist, L.; Kitchin, J. R.; Bligaard, T.; Jonsson, H. Origin of the Overpotential for Oxygen Reduction at a Fuel-Cell Cathode. The Journal of Physical Chemistry B 2004, 108, 17886-17892. 
(49) Chen, L. D.; Urushihara, M.; Chan, K.; Nørskov, J. K. Electric Field Effects in Electrochemical CO2 Reduction. ACS Catalysis 2016, 6, 7133-7139.

(50) Montoya, J. H.; Shi, C.; Chan, K.; Nørskov, J. K. Theoretical Insights Into a CO Dimerization Mechanism in CO2 Electroreduction. The journal of physical chemistry letters 2015, 6, 2032-2037.

(51) Sandberg, R. B.; Montoya, J. H.; Chan, K.; Nørskov, J. K. CO-CO Coupling on Cu Facets: Coverage, Strain and Field Effects. Surface Science 2016, 654, 56-62.

(52) Chan, K.; Nørskov, J. K. Electrochemical Barriers Made Simple. The Journal of Physical Chemistry Letters 2015, 6, 2663-2668, PMID: 26266844.

(53) Skúlason, E.; Karlberg, G. S.; Rossmeisl, J.; Bligaard, T.; Greeley, J.; Jónsson, H.; Nørskov, J. K. Density Functional Theory Calculations for the Hydrogen Evolution Reaction in an Electrochemical Double Layer on the Pt (111) Electrode. Physical Chemistry Chemical Physics 2007, 9, 3241-3250.

(54) Karlberg, G.; Rossmeisl, J.; Nørskov, J. K. Estimations of Electric Field Effects on the Oxygen Reduction Reaction Based on the Density Functional Theory. Physical Chemistry Chemical Physics 2007, 9, 5158-5161.

(55) Skúlason, E.; Tripkovic, V.; Bjorketun, M. E.; Gudmundsdottir, S.; Karlberg, G.; Rossmeisl, J.; Bligaard, T.; Jónsson, H.; Nørskov, J. K. Modeling the Electrochemical Hydrogen Oxidation and Evolution Reactions on the Basis of Density Functional Theory Calculations. The Journal of Physical Chemistry C 2010, 114, 18182-18197.

(56) Mattioli, G.; Giannozzi, P.; Amore Bonapasta, A.; Guidoni, L. Reaction Pathways for Oxygen Evolution Promoted by Cobalt Catalyst. Journal of the American Chemical Society 2013, 135, 15353-15363. 
(57) Liu, X.; Xiao, J.; Peng, H.; Hong, X.; Chan, K.; Nørskov, J. K. Understanding Trends in Electrochemical Carbon Dioxide Reduction Rates. Nature Communications 2017, 8, 15438.

(58) Hussain, J.; Jonsson, H.; Skúlason, E. Calculations of Product Selectivity in Electrochemical CO2 Reduction. ACS Catalysis 2018,

(59) Cheng, T.; Xiao, H.; Goddard, W. A. Full Atomistic Reaction Mechanism With Kinetics for CO Reduction on $\mathrm{Cu}$ (100) From Ab Initio Molecular Dynamics FreeEnergy Calculations at 298 K. Proceedings of the National Academy of Sciences 2017, 201612106.

(60) Huang, B.; Muy, S.; Feng, S.; Katayama, Y.; Lu, Y.-C.; Chen, G.; Yang, S.-H. NonCovalent Interactions in Electrochemical Reactions and Implications for Clean Energy Applications. Physical Chemistry Chemical Physics 2018,

(61) Dinh, C.-T.; Burdyny, T.; Kibria, M. G.; Seifitokaldani, A.; Gabardo, C. M.; García de Arquer, F. P.; Kiani, A.; Edwards, J. P.; De Luna, P.; Bushuyev, O. S. et al. CO2 Electroreduction to Ethylene via Hydroxide-Mediated Copper Catalysis at an Abrupt Interface. Science 2018, 360, 783-787.

(62) Lum, Y.; Cheng, T.; Goddard III, W. A.; Ager, J. W. Electrochemical CO Reduction Builds Solvent Water Into Oxygenate Products. Journal of the American Chemical Society 2018, 140, 9337-9340.

(63) Jinnouchi, R.; Nagoya, A.; Kodama, K.; Morimoto, Y. Solvation Effects on OH Adsorbates on Stepped Pt Surfaces. The Journal of Physical Chemistry C 2015, 119, 16743-16753.

(64) Michel, C.; Zaffran, J.; Ruppert, A. M.; Matras-Michalska, J.; Jdrzejczyk, M.; Grams, J.; Sautet, P. Role of Water in Metal Catalyst Performance for Ketone Hydro- 
genation: A Joint Experimental and Theoretical Study on Levulinic Acid Conversion Into Gamma-Valerolactone. Chem. Commun. 2014, 50, 12450-12453.

(65) Zaffran, J.; Michel, C.; Delbecq, F.; Sautet, P. Towards More Accurate Prediction of Activation Energies for Polyalcohol Dehydrogenation on Transition Metal Catalysts in Water. Catalysis Science \& Technology 2016, 6, 6615-6624.

(66) Bagger, A.; Arnarson, L.; Hansen, M. H.; Spohr, E.; Rossmeisl, J. Electrochemical CO Reduction: A property of the electrochemical interface. Journal of the American Chemical Society 2019,

(67) Limmer, D. T.; Willard, A. P.; Madden, P. A.; Chandler, D. Water Exchange at a Hydrated Platinum Electrode Is Rare and Collective. The Journal of Physical Chemistry C 2015, 119, 24016-24024.

(68) Limmer, D. T.; Willard, A. P.; Madden, P.; Chandler, D. Hydration of Metal Surfaces Can Be Dynamically Heterogeneous and Hydrophobic. Proceedings of the National Academy of Sciences 2013, 110, 4200-4205.

(69) Siepmann, J. I.; Sprik, M. Influence of Surface Topology and Electrostatic Potential on Water/Electrode Systems. The Journal of chemical physics 1995, 102, 511-524.

(70) Willard, A. P.; Reed, S. K.; Madden, P. A.; Chandler, D. Water at an Electrochemical Interface - a Simulation Study. Faraday discussions 2009, 141, 423-441.

(71) Steinmann, S. N.; Ferreira de Morais, R.; Götz, A. W.; Fleurat-Lessard, P.; Iannuzzi, M.; Sautet, P.; Michel, C. A Force Field for Water Over Pt (111): Development, Assessment and Comparison. Journal of chemical theory and computation 2018,

(72) Petrosyan, S.; Briere, J.-F.; Roundy, D.; Arias, T. Joint Density-Functional Theory for Electronic Structure of Solvated Systems. Physical Review B 2007, 75, 205105. 
(73) Nishihara, S.; Otani, M. Hybrid Solvation Models for Bulk, Interface, and Membrane: Reference Interaction Site Methods Coupled With Density Functional Theory. Physical Review B 2017, 96, 115429.

(74) Andreussi, O.; Dabo, I.; Marzari, N. Revised Self-Consistent Continuum Solvation in Electronic-Structure Calculations. The Journal of chemical physics 2012, 136, 064102.

(75) Steinmann, S. N.; Sautet, P.; Michel, C. Solvation Free Energies for Periodic Surfaces: Comparison of Implicit and Explicit Solvation Models. Physical Chemistry Chemical Physics 2016, 18, 31850-31861.

(76) Steinmann, S. N.; Michel, C.; Schwiedernoch, R.; Sautet, P. Impacts of Electrode Potentials and Solvents on the Electroreduction of CO2: A Comparison of Theoretical Approaches. Physical Chemistry Chemical Physics 2015, 17, 13949-13963.

(77) Gauthier, J. A.; Ringe, S.; Dickens, C. F.; Garza, A. J.; Bell, A. T.; Head-Gordon, M.; Nørskov, J. K.; Chan, K. Challenges in Modeling Electrochemical Reaction Energetics with Polarizable Continuum Models. ACS Catalysis 2018, 9, 920-931.

(78) Montoya, J. H.; Peterson, A. A.; Nørskov, J. K. Insights Into C-C Coupling in CO2 Electroreduction on Copper Electrodes. ChemCatChem 2013, 5, 737-742.

(79) Goedecker, S. Minima Hopping: An Efficient Search Method for the Global Minimum of the Potential Energy Surface of Complex Molecular Systems. The Journal of Chemical Physics 2004, 120, 9911-9917.

(80) Peterson, A. A. Global Optimization of Adsorbate-Surface Structures While Preserving Molecular Identity. Topics in Catalysis 2014, 57, 40-53.

(81) Giannozzi, P.; Baroni, S.; Bonini, N.; Calandra, M.; Car, R.; Cavazzoni, C.; Ceresoli, D.; Chiarotti, G. L.; Cococcioni, M.; Dabo, I. et al. QUANTUM ESPRESSO: 
A Modular and Open-Source Software Project for Quantum Simulations of Materials. Journal of Physics: Condensed Matter 2009, 21, 395502.

(82) Larsen, A. H.; Mortensen, J. J.; Blomqvist, J.; Castelli, I. E.; Christensen, R.; Duak, M.; Friis, J.; Groves, M. N.; Hammer, B.; Hargus, C. et al. The Atomic Simulation Environment - a Python Library for Working with Atoms. Journal of Physics: Condensed Matter 2017, 29, 273002.

(83) Wellendorff, J.; Lundgaard, K. T.; Møgelhøj, A.; Petzold, V.; Landis, D. D.; Nørskov, J. K.; Bligaard, T.; Jacobsen, K. W. Density Functionals for Surface Science: Exchange-Correlation Model Development With Bayesian Error Estimation. Phys. Rev. B 2012, 85, 235149.

(84) Dion, M.; Rydberg, H.; Schröder, E.; Langreth, D. C.; Lundqvist, B. I. Van Der Waals Density Functional for General Geometries. Phys. Rev. Lett. 2004, 92, 246401.

(85) Thonhauser, T.; Cooper, V. R.; Li, S.; Puzder, A.; Hyldgaard, P.; Langreth, D. C. Van Der Waals Density Functional: Self-Consistent Potential and the Nature of the Van Der Waals Bond. Phys. Rev. B 2007, 76, 125112.

(86) Román-Pérez, G.; Soler, J. M. Efficient Implementation of a Van Der Waals Density Functional: Application to Double-Wall Carbon Nanotubes. Phys. Rev. Lett. 2009, 103, 096102.

(87) Vanderbilt, D. Soft Self-Consistent Pseudopotentials in a Generalized Eigenvalue Formalism. Phys. Rev. B 1990, 41, 7892-7895.

(88) Laasonen, K.; Car, R.; Lee, C.; Vanderbilt, D. Implementation of Ultrasoft Pseudopotentials in Ab Initio Molecular Dynamics. Phys. Rev. B 1991, 43, 6796-6799.

(89) Laasonen, K.; Pasquarello, A.; Car, R.; Lee, C.; Vanderbilt, D. Car-Parrinello Molec- 
ular Dynamics With Vanderbilt Ultrasoft Pseudopotentials. Phys. Rev. B 1993, 47, 10142-10153.

(90) Garrity, K. F.; Bennett, J. W.; Rabe, K. M.; Vanderbilt, D. Pseudopotentials for High-Throughput DFT Calculations. Computational Materials Science 2014, 81, 446 $-452$.

(91) Monkhorst, H. J.; Pack, J. D. Special Points for Brillouin-Zone Integrations. Phys. Rev. B 1976, 13, 5188-5192.

(92) Stillinger, F. H. Exponential Multiplicity of Inherent Structures. Physical Review E 1999, 59, 48.

(93) Manz, T. A. Introducing DDEC6 Atomic Population Analysis: Part 3. Comprehensive Method to Compute Bond Orders. RSC Advances 2017, 7, 45552-45581.

(94) Limas, N. G.; Manz, T. A. Introducing DDEC6 Atomic Population Analysis: Part 2. Computed Results for a Wide Range of Periodic and Nonperiodic Materials. $R S C$ Advances 2016, 6, 45727-45747.

(95) Mortensen, J. J.; Hansen, L. B.; Jacobsen, K. W. Real-Space Grid Implementation of the Projector Augmented Wave Method. Physical Review B 2005, 71, 035109.

(96) Enkovaara, J.; Rostgaard, C.; Mortensen, J. J.; Chen, J.; Duak, M.; Ferrighi, L.; Gavnholt, J.; Glinsvad, C.; Haikola, V.; Hansen, H. A. et al. Electronic Structure Calculations With GPAW: A Real-Space Implementation of the Projector AugmentedWave Method. Journal of Physics: Condensed Matter 2010, 22, 253202.

(97) Humphrey, W.; Dalke, A.; Schulten, K. VMD - Visual Molecular Dynamics. Journal of Molecular Graphics 1996, 14, 33-38. 
(98) Tripkovic, V.; Björketun, M. E.; Skúlason, E.; Rossmeisl, J. Standard Hydrogen Electrode and Potential of Zero Charge in Density Functional Calculations. Physical Review B 2011, 84, 115452.

(99) Materzanini, G.; Tantardini, G. F.; Lindan, P. J.; Saalfrank, P. Water Adsorption at Metal Surfaces: A First-Principles Study of the p $(3 \times 3)$ R 30 H2O Bilayer on Ru (0001). Physical Review B 2005, 71, 155414.

(100) Jinnouchi, R.; Anderson, A. B. Electronic Structure Calculations of Liquid-Solid Interfaces: Combination of Density Functional Theory and Modified Poisson-Boltzmann Theory. Physical Review B 2008, 77, 245417.

(101) Quaranta, V.; Hellstrom, M.; Behler, J. Proton-Transfer Mechanisms at the WaterZnO Interface: The Role of Presolvation. The Journal of Physical Chemistry Letters 2017, 8, 1476-1483.

(102) Biswas, R.; Tse, Y.-L. S.; Tokmakoff, A.; Voth, G. A. Role of Presolvation and Anharmonicity in Aqueous Phase Hydrated Proton Solvation and Transport. The Journal of Physical Chemistry B 2015, 120, 1793-1804.

(103) Ando, K.; Hynes, J. T. Molecular Mechanism of $\mathrm{HCl}$ Acid Ionization in Water: Ab Initio Potential Energy Surfaces and Monte Carlo Simulations. The Journal of Physical Chemistry B 1997, 101, 10464-10478.

(104) Marcus, R. A. Chemical and Electrochemical Electron-Transfer Theory. Annual review of physical chemistry 1964, 15, 155-196.

(105) Trasatti, S. The Absolute Electrode Potential: An Explanatory Note. International Union of Pure and Applied Chemistry 1986, 58, 955-966.

(106) Gaiduk, A. P.; Gustafson, J. A.; Gygi, F.; Galli, G. First-Principles Simulations of 
Liquid Water Using a Dielectric-Dependent Hybrid Functional. The journal of physical chemistry letters 2018,

(107) Gaiduk, A. P.; Pham, T. A.; Govoni, M.; Paesani, F.; Galli, G. Electron Affinity of Liquid Water. Nature communications 2018, 9, 247.

(108) Gillan, M. J.; Alfè, D.; Michaelides, A. Perspective: How Good Is DFT for Water? The Journal of chemical physics 2016, 144, 130901.

(109) Sun, J.; Remsing, R. C.; Zhang, Y.; Sun, Z.; Ruzsinszky, A.; Peng, H.; Yang, Z.; Paul, A.; Waghmare, U.; Wu, X. et al. Accurate First-Principles Structures and Energies of Diversely Bonded Systems From an Efficient Density Functional. Nature chemistry 2016, 8, 831.

(110) Dawson, W.; Gygi, F. Equilibration and Analysis of First-Principles Molecular Dynamics Simulations of Water. The Journal of chemical physics 2018, 148, 124501. 
Graphical TOC Entry

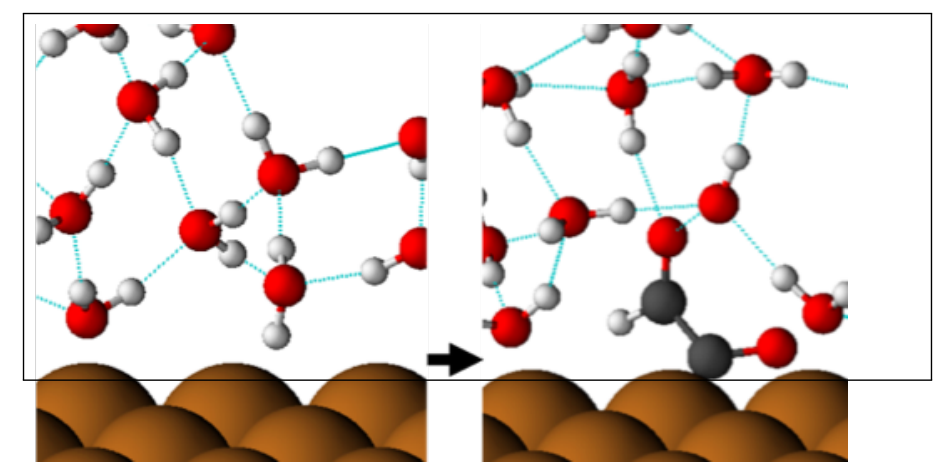

